\title{
Estimates of the Prevalence of Tobacco Smoking in Egypt
}

\author{
Christopher A. Loffredo1 ${ }^{*}$, Ghada Nasr Radwan'2, Eman M. Eltahlawy ${ }^{3}$, Maged El-Setouhy4,5, \\ Laurence Magder6, Mohamed Hassan Hussein ${ }^{2}$ \\ ${ }^{1}$ Georgetown University, Washington DC, USA \\ ${ }^{2}$ Cairo University, Cairo, Egypt \\ ${ }^{3}$ National Research Center, Cairo, Egypt \\ ${ }^{4}$ Jazan University, Jazan, Saudi Arabia \\ ${ }^{5}$ Ain Shams University, Cairo, Egypt \\ ${ }^{6}$ University of Maryland, Baltimore, USA \\ Email: *al9@georgetown.edu
}

Received 7 April 2015; accepted 7 May 2015; accepted 12 May 2015

Copyright (C) 2015 by authors and Scientific Research Publishing Inc.

This work is licensed under the Creative Commons Attribution International License (CC BY).

http://creativecommons.org/licenses/by/4.0/

(c) (i) Open Access

\section{Abstract}

The use of tobacco products constitutes the leading cause of preventable deaths in the world. In Egypt, data on the national prevalence of cigarette and waterpipe smoking are lacking, yet such data are vital to tobacco control efforts. We designed and implemented a sampling method to generate population-based prevalence estimates for tobacco smoking in Egypt. In 2005, a total of 3369 adults ages 18 and above, including 1867 females and 1502 males, participated in the survey. Among males, the national prevalence of former cigarette smoking was $18.1 \%$, and $27.5 \%$ reported current smoking. Exclusive current waterpipe smoking was reported by $7.2 \%$ of adult males, and a further 3.4\% reported smoking both waterpipes and cigarettes. Tobacco smoking was rarely reported by females; among them the national prevalence of current cigarette smoking was $0.3 \%$. This study produced the first robust estimate of the national prevalence of cigarette and waterpipe smoking in Egypt based on a rigorous probability sample. Along with the more recent Global Adult Tobacco Survey, carried out by the World Health Organization in 2009, the results will be useful in assessing smoking trends in Egypt and in evaluating the impacts of recent tobacco control laws.

\section{Keywords}

Egypt, Smoking, Tobacco, Waterpipe, Shisha, Survey

${ }^{*}$ Corresponding author.

How to cite this paper: Loffredo, C.A., Radwan, G.N., Eltahlawy, E.M., El-Setouhy, M., Magder, L. and Hussein, M.H. (2015) Estimates of the Prevalence of Tobacco Smoking in Egypt. Open Journal of Epidemiology, 5, 129-135. 


\section{Introduction}

The use of tobacco products constitutes the leading cause of preventable deaths in the world. To address this global epidemic, an international framework for tobacco control was initiated in 1995. On May 21, 2003, a landmark date for public health, the 192 member states of the 56th World Health Assembly unanimously adopted the world's first public health treaty, the Framework Convention on Tobacco Control [1].

In order to fully implement the Framework Convention on Tobacco Control and assess trends in use, governments require reliable data on the prevalence of all forms of tobacco consumption. In Egypt, as in many countries, effective tobacco control and prevention policies have been hindered by a lack of reliable statistics on the prevalence of smoking practices. Until the Global Adult Tobacco Survey in 2009 [2], no national prevalence data were available in Egypt, and thus there was a major gap in knowledge regarding smoking prevalence at the time of our survey in 2005. In addition, smoking rates may vary between population subgroups and between males and females as a result of cultural norms regarding smoking behaviors in Egypt [3], and it is important to explore these variations. There are also possible socio-cultural differences in smoking behaviors between urban and rural areas. Finally, Egypt is well known for the use of waterpipes (shisha) for tobacco smoking [4], and at the time of the study national prevalence estimates were almost entirely lacking for this method of smoking. Therefore, our study aimed to produce the first robust estimates of the national prevalence of cigarette and waterpipe smoking in Egypt.

\section{Materials and Methods}

This study was designed to provide an assessment of the prevalence of cigarette smoking and waterpipe smoking among adult males and females (age 18 and above) in the general population of Egypt in 2005. We used a multi-stage probability strategy to obtain a sample of individuals who self-reported their cigarette and waterpipe (shisha) smoking habits.

The 323 districts of Egypt defined by the Central Agency for Public Mobilization and Statistics were divided into six strata based on geography. In the first stage of the survey, we randomly sampled $20 \%$ of the districts within each stratum, yielding 65 primary sampling units. Each district is divided by the Central Agency for Public Mobilization and Statistics into villages in rural areas and into police districts in urban areas. We randomly selected two villages or police districts and within each sampled village or police district, two streets were randomly chosen. From each street seven or eight buildings were systematically sampled. Within each building, a single household was randomly selected, from which one household was randomly chosen, and within each household one adult male and one adult female were randomly selected. Once the survey team identified the sampled individuals, they were invited to participate in the survey. For all stages, the random number generator function of MS Excel was used to provide tables of random numbers.

Using the questionnaire responses (see Appendix for the survey instrument), individuals were categorized as never-smokers, former smokers, current smokers, or experimental smokers of cigarettes. Never smokers were defined as those who had never smoked cigarettes in their lifetime. Experimental smokers were those who had smoked more than one but less than 100 cigarettes in their lifetime. Those who had smoked at least 100 cigarettes in their lifetime were categorized as either former or current smokers [5]. Former smokers were those who had not smoked during the last month, while current smokers had smoked in the past 15 days. For some analyses we broadened the definition of current smokers to include those who had smoked in the past 30 days. Separate questions were asked about waterpipe smoking, from which the respondents were categorized as never, experimental, former, or current waterpipes smokers, using the cutoff point of smoking in the past 30 days to distinguish between former and current waterpipes smokers. Using weights based on the sample design, we obtained the weighted prevalence point estimate and its 95\% confidence interval (CI) for each type of smoking behavior, for males and females separately and for urban and rural areas separately. This study was approved by the Institutional Review Boards of the University of Maryland, Baltimore, and the Ministry of Health and Population of Egypt. Written informed consent was obtained from all participants.

\section{Results}

\subsection{Study Population}

The implementation of this sampling method resulted from field work conducted in 65 (20\%) of the 323 admin- 
istrative districts of Egypt and produced a list of 3613 adults who were scheduled to be contacted. Of these, 174 (3.8\%) were not at home when the survey team arrived and also missed the repeat visit, 10 subjects $(<1 \%)$ who were contacted refused to participate, and a further 130 subjects (2.8\%) were excluded due to incomplete data. A total of 1938 households participated, of which 334 (17\%) contributed only one respondent. The 3369 adult respondents with complete data were included in the statistical analyses. This included 1502 males and 1867 females, with median ages of 40 and 37, respectively. The overall participation rate was $93.2 \%$.

\subsection{Prevalence of Cigarette Smoking in Males}

Among males, our results estimated a national prevalence of $46.9 \%$ (95\% CI 41.8 - 52.1) never smokers at the time of the study (Table 1). Experimental cigarette smokers had a national prevalence of 7.4\% (95\% CI 5.1 10.8), and former smokers had a national prevalence $18.1 \%$ (95\% CI 14.2 - 22.9). Current cigarette smoking (in the past 15 days) was practiced by $27.5 \%$ of males (95\% CI 23.4 - 31.9). Since males with a low income may not have been able to afford daily smoking, we also revised the definition of current smoking to include cigarette smoking in the past 30 days, which may have more accurately reflected smoking behavior in this group [6]. The revised definition yielded an estimate of $31.1 \%$ males currently smoking cigarettes (data not shown) at the time of the study (95\% CI 26.5 - 36.0).

\subsection{Prevalence of Cigarette Smoking in Females}

Cigarette smoking was rarely reported by females: our results estimated a national prevalence of $97 \%$ for never smokers (95\% CI 96.9 - 98.8), a prevalence of $1.6 \%$ for experimental smokers (95\% CI 0.8 - 2.3), and $0.8 \%$ for former smokers (95\% CI 0.1 - 0.9) (Table 1). For females we used the broader definition of current smoking (in the past 30 days), which, as in males, may have more accurately reflected smoking behavior in Egypt [6]. According to this definition, current cigarette smoking was practiced by $0.3 \%$ of females (95\% CI $0.1-1.1$ ).

\subsection{Urban and Rural Areas}

Cigarette smoking prevalence was similar between urban and rural populations: the prevalence of never smokers among males was $47.3 \%$ in rural areas compared to $46.6 \%$ in urban areas, and $17.9 \%$ and $18.4 \%$ were former smokers, respectively. Current cigarette smoking was practiced by $28.6 \%$ (95\% CI 23.1 - 34.9) of rural males vs.

Table 1. Prevalence of cigarette smoking in Egypt among adult males and females in 2005.

\begin{tabular}{ccccc}
\hline \multirow{2}{*}{ Adult males } & \multicolumn{3}{c}{ Prevalence } \\
\cline { 2 - 5 } Never smoker & 667 & Percent & \multicolumn{3}{c}{$95 \% \mathrm{CI}$} \\
Experimental smoker & 95 & 46.9 & 41.8 & 52.1 \\
Former smoker & 271 & 7.4 & 5.1 & 10.8 \\
Current smoker & 469 & 18.1 & 14.2 & 22.9 \\
Total & 1502 & 27.5 & 23.4 & 31.9 \\
Adult females & & & & \\
Never smoker & 1817 & 97 & 96.9 & 98.8 \\
Experimental smoker & 30 & 1.6 & 0.8 & 2.3 \\
Former smoker & 15 & 0.8 & 0.1 & 0.9 \\
Current smoker & 5 & 0.3 & 0.1 & \\
Total & 1867 & & & \\
\hline
\end{tabular}

*Defined as those who had smoked at least 100 cigarettes in their lifetime and had smoked in the past 15 days; *** Defined as those who had smoked at least 100 cigarettes in their lifetime and had smoked in the past 30 days. 
26.3\% of urban males (95\% CI 20.7 - 32.9). Similarly, there were no significant urban-rural differences in smoking prevalence among adult females.

\subsection{Age of Initiation of Cigarette Smoking}

Survey respondents were asked to report the age at which they first tried smoking cigarettes. Among males who had ever tried smoking, $60 \%$ were aged 15 years or younger, and $88 \%$ had tried it by age 20 . Among females the age at initiation shifted slightly to the older age categories, with $60 \%$ initiating by age 20 and $87 \%$ by age 25 .

\subsection{Waterpipe (Shisha) Smoking}

A total of 84 males (5.6\%) reported exclusively smoking shisha currently, while $16.9 \%$ were exclusive cigarette smokers at the time of the survey. Current users of both smoking methods comprised $4.1 \%$ of the respondents. The remainder (20.5\%) were experimental and former smokers of cigarettes or shisha. Over one third of males (36.8\%) never smoked cigarettes or waterpipes. Table 2 shows the joint distributions of cigarette smoking and waterpipe smoking in the sample of males. The number of females $(\mathrm{N}=5)$ who reported using waterpipes was too small to permit meaningful analysis.

\section{Discussion}

We designed and implemented this sampling method to obtain a random, stratified sample of individuals to be interviewed in their homes regarding their tobacco smoking habits. The survey was designed to generate robust estimates of the national prevalence of these behaviors among adults in Egypt in 2005. For adult males, the prevalence of current cigarette smoking was $27.5 \%$ (defined as smoking in the past 15 days) and $31.1 \%$ (defined as smoking in the past 30 days). The 15-day metric corresponds to the stricter definition of smoking, frequently used in other research. The 30-day metric may more accurately reflect the range of typical smoking behavior in Egypt, because it includes numerous non-daily smokers [6]. These prevalence estimates predict a total population of 5 - 8 million adult male cigarette smokers based on the 2005 population, while another 1.9 million were currently smoking waterpipes. In contrast, very few adult women reported that they were current or former smokers of any type of tobacco product.

In 2005, at the time of the study, no national prevalence surveys of smoking in Egypt had been published. A

Table 2. Waterpipe smoking in relation to cigarette smoking among males in Egypt, 2005.

\begin{tabular}{lcc}
\hline & Number & (\%) \\
\hline Used neither type of tobacco & 553 & $\mathbf{3 6 . 8}$ \\
Exclusive cigarette smokers & & \\
$\quad$ Experimental or former smoker & 147 & 9.8 \\
Current smoker & 295 & 19.7 \\
Exclusive waterpipe smokers & & \\
Experimental or former smoker & 30 & 2.0 \\
Current smoker & 84 & 5.6 \\
Smokers of both products & 140 & \\
$\quad$ Experimental or former smoker & 71 & 9.3 \\
Currently smoking both types & 139 & 4.7 \\
Current cigarette, former waterpipe & 42 & 9.3 \\
Current waterpipe, former cigarette & 1501 & 100 \\
\hline
\end{tabular}


systematic survey of the general population in 2009, the Global Adult Tobacco Survey (GATS), found that 37.7\% of the Egyptian male population used some form of tobacco product [2], whereas our study found the prevalence of current smoking among adult males to be $27.5 \%$ in 2005 . The lower smoking prevalence reported in this study among adult males compared to the results in GATS might be attributed to different definitions of current smoking in the two surveys. The GATS definition relied on reporting of smoking on a daily basis regardless of whether participants had smoked 100 cigarettes in their lifetime, whereas we defined current smokers as those who had smoked at least 100 cigarettes in their lifetime and had smoked in the past 30 days. Our study found a similar prevalence of smoking among females to that found in GATS - with $0.6 \%$ of the Egyptian female population having reported use of some form of tobacco in GATS, compared to $0.3 \%$ of females reporting smoking cigarettes in our survey (the number of females who reported using waterpipes in our survey was too small to permit meaningful analysis.)

Several studies had estimated smoking prevalence in specific subgroups at the time of our study in 2005. The Global Health Professionals Survey reported that among 1770 third year medical students from 14 Egyptian medical schools, $12.9 \%$ of males and $1.2 \%$ of females were current smokers of cigarettes, and an additional $11.9 \%$ and $1.4 \%$, respectively, were currently smoking water pipes [7]. In a convenience sample of 2120 persons ages 15 - 86 years in the general population of the city of Alexandria, Egypt, the overall prevalence of current tobacco smoking of all types combined was $48.5 \%$ among males and $1.5 \%$ among females [8]. Three other studies in Egypt have yielded data on group waterpipe smoking among males. A study in 2001 of a community in the Nile Delta found that $10.6 \%$ of males reported group waterpipe smoking [9]. A study in 2002 in a community in Upper Egypt found that 13\% of males reported group waterpipe smoking [10]. Finally, a study in 2004 in the Sharkia Governorate found that $14.6 \%$ of males reported group waterpipe smoking [11].

One of the challenges in characterizing tobacco smoking in low resource countries, including Egypt, is the lack of consistent definitions of smoking behaviors and patterns in published reports, especially regarding smoking among adults. Moreover, waterpipe smoking has rarely been queried or reported in studies appearing in the international peer-reviewed literature. This situation is in marked contrast to the Global Adult Tobacco Survey, which included cross-country comparisons of smoking behavior based on the application of consistent definitions and comparable survey methods.

Estimations of tobacco smoking prevalence in the general population of adults in the Middle East and North Africa have mostly been extrapolated from convenience sampling. Patients attending health care facilities are one example of the use of this sampling approach in studies of tobacco use. In a study of 1534 adult subjects attending primary health facilities in Saudi Arabia, the proportions of current and former smokers were $25.3 \%$ and $10.2 \%$, respectively [12]. In another study of 634 subjects in that country, 34.4\% self-reported current smoking and a further $16.4 \%$ reported former smoking [13]. In Jordan, a study of 5000 persons with coronary heart disease in screening clinics reported a prevalence of tobacco smoking of $45 \%$ among men and $11 \%$ among women [14]. The above studies would likely over-estimate the national prevalence of tobacco smoking in their respective countries due to selection bias, as smokers may be more likely than non-smokers to seek health care for smoking-related diseases.

Alternative study designs have included surveys of non-treatment seeking individuals. In Saudi Arabia, for example, parents of schoolboys were surveyed regarding tobacco smoking; the study found that $32 \%$ of fathers and $4 \%$ of mothers of 1482 boys (ages 6 - 15 years) reported smoking [15]. Also in Saudi Arabia, a household-based survey on tobacco smoking habits using a stratified cluster sampling technique found that $21.1 \%$ of males and $0.9 \%$ of females reported currently cigarette smoking [16]. In Jordan, questions about tobacco smoking and other behavioral risk factors for chronic diseases were included in a national, cross-sectional employment survey of adults in 2002, using a national sampling frame; the results revealed that $51 \%$ of males and $8 \%$ of females reported currently smoking cigarettes [17]. In Lebanon, a study was carried out via a multilevel cluster sampling technique, stratified by district, and the authors reported an adult smoking prevalence of 53.6\% [18]. A household survey carried out in urban areas in Sousse, Tunisia, using a two-stage systematic sampling method reported that $61.4 \%$ of adult males and $4.2 \%$ of adult females reported smoking [19]. It is unclear whether these highly variable results across countries reflect cultural differences, variations in sampling methods and definitions of smoking, or differences in tobacco prevention policies and programs.

Among the limitations of our study was the small number of self-reported adult female smokers. This may have been because women might not have felt free to discuss their tobacco smoking habits during a household-based interview, in comparison to other venues such as waterpipe cafes [3]. 


\section{Conclusion}

This study, based on data from 2005, produced the first estimates of the national prevalence of cigarette and waterpipe smoking in the general population of Egypt obtained from a stratified random sample with a high participation rate. Along with the more recent Global Adult Tobacco Survey, carried out by the World Health Organization in 2009, these results are important for tobacco control efforts at the national and local levels, particularly as the public sector in Egypt continues to design and implement policies to enforce tobacco control laws. One of the major implications of our findings is the strong likelihood that, due to the high prevalence of adult male cigarette and waterpipe smoking, levels of environmental tobacco smoke are likely to be very high in homes and workplaces. As a result, many women, children, and other non-smokers are likely being exposed. This is supported by the results of the Global Youth Tobacco Survey in Egypt, in which 38.0\% of the participating children reported being exposed to environmental tobacco smoke at home and 52.9\% reported living with at least one parent who smoked [20]. Other results from Global Youth Tobacco Surveys in the region have reported similar findings, ranging from $40.3 \%$ of children being exposed to environmental tobacco smoke at home in Libya [21] to $78.9 \%$ in Lebanon [22]. Moreover, recent projections for tobacco smoking rates have predicted rapid increases among both men and women in the Eastern Mediterranean region, pointing to the need for enhanced measures for tobacco control [23]. The Egyptian government in particular can contribute to tobacco control by continuing to implement policies and programs in line with the Framework Convention on Tobacco Control, with a particular focus on enforcement.

\section{Acknowledgements}

This work was supported by grant number R01TW05944 from the Fogarty International Center, U.S. National Institutes of Health. The authors thank Nabiel N. Mikhail for data management. Important contributions were also made by the late Dr. Mostafa K. Mohamed, to whom this manuscript is dedicated. Additionally, we thank Maha Maher for coordinating and supervising the extensive fieldwork for this study.

\section{References}

[1] World Health Organization (2003) An International Treaty for Tobacco Control.

[2] World Health Organization Regional Office for the Eastern Mediterranean (2009) Global Adult Tobacco Survey: Egypt.

[3] Labib, N., Radwan, G., Mikhail, N., Mohamed, M.K., El Setouhy, M., Loffredo, C. and Israel, E. (2007) Comparison of Cigarette and Waterpipe Smoking among Female University Students in Egypt. Nicotine \& Tobacco Research, 9, 591-596. http://dx.doi.org/10.1080/14622200701239696

[4] World Health Organization Regional Office for the Eastern Mediterranean (2006) The Health Hazards of Smoking Shisha.

[5] Centers for Disease Control and Prevention (2005) Cigarette Smoking Among Adults. MMWR, 54, 509-513.

[6] Boulos, D.N., Loffredo, C.A., El Setouhy, M., Abdel-Aziz, F., Israel, E. and Mohamed, M.K. (2009) Nondaily, Light Daily, and Moderate-to-Heavy Cigarette Smokers in a Rural Area of Egypt: A Population-Based Survey. Nicotine \& Tobacco Research, 11, 134-138. http://dx.doi.org/10.1093/ntr/ntp016

[7] Centers for Disease Control and Prevention (2005) Global Health Professionals Survey: Egypt.

[8] Youssef, R.M., Abou-Khatwa, S.A. and Fouad, H.M. (2002) Prevalence of Smoking and Age of Initiation in Alexandria, Egypt. Eastern Mediterranean Health Journal, 8, 626-637.

[9] Habib, M., Mohamed, M.K., Abdel-Aziz, F., Magder, L.S., Abdel-Hamid, M., Gamil, F., Madkour, S., Mikhail, N.N., Anwar, W., Strickland, G.T., Fix, A.D. and Sallam, I. (2001) Hepatitis C Virus Infection in a Community in the Nile Delta: Risk Factors for Seropositivity. Hepatology, 33, 248-253. http://dx.doi.org/10.1053/jhep.2001.20797

[10] Medhat, A., Shehata, M., Magder, L.S., Mikhail, N., Abdel-Baki, L., Nafeh, M., Abdel-Hamid, M., Strickland, G.T. and Fix, A.D. (2002) Hepatitis C in a Community in Upper Egypt: Risk Factors for Infection. The American Journal of Tropical Medicine and Hygiene, 66, 633-638.

[11] El-Sadawy, M., Ragab, H., El-Toukhy, H., El-Mor, A.-L., Mangoud, A.M., Eissa, M.H., Afefy, A.F., El-Shorbagy, E., Ibrahem, I.A., Mahrous, S., Abdel-Monem, A., Sabee, E.I., Ismail, A., Morsy, T.A., Etewa, S., Nor Edin, E., Mostafa, Y., Abouel-Magd, Y., Hassan, M.I., Lakouz, K., Abdel-Aziz, K., El-Hady, G. and Saber, M. (2004) Hepatitis C Virus Infection at Sharkia Governorate, Egypt: Seroprevalence and Associated Risk Factors. Journal of the Egyptian Society of Parasitology, 34, 367-384. 
[12] Saeed, A.A., Khoja, T.A. and Khan, S.B. (1996) Smoking Behavior and Attitudes among Saudi Nationals in Riyadh City, Saudi Arabia. Tobacco Control, 5, 215-219. http://dx.doi.org/10.1136/tc.5.3.215

[13] Siddiqui, S., Ogbeide, D.O. and Al Khalifa, I. (2001) Smoking in a Saudi Community: Prevalence, Influencing Factors, and Risk Perception. Family Medicine, 33, 367-370.

[14] Hammoudeh, A.J., Al-Tarawneh, H., Elharassis, A., Haddad, J., Mahadeen, Z., Badran, N., Izraiq, M. and Al-Mousa, E. (2006) Prevalence of Conventional Risk Factors in Jordanians with Coronary Heart Disease: The Jordan Hyperlipidemia and Related Targets Study (JoHARTS). International Journal of Cardiology, 110, 179-183. http://dx.doi.org/10.1016/j.ijcard.2005.08.005

[15] Al-Dawood, K.M. (2000) Pattern of Smoking among Parents of Schoolboys. Saudi Medical Journal, 21, 735-739.

[16] Jarallah, J.S., Al-Rubeaan, K.A., Al-Nuaim, A.R., Al-Ruhaily, A.A. and Kalantan, K.A. (1999) Prevalence and Determinants of Smoking in Three Regions of Saudi Arabia. Tobacco Control, 8, 53-56. http://dx.doi.org/10.1136/tc.8.1.53

[17] Centers for Disease Control and Prevention (2003) Prevalence of Selected Risk Factors for Chronic Disease-Jordan, 2002. MMWR, 52, 1042-1044.

[18] Baddoura, R. and Wehbeh-Chidiac, C. (2001) Prevalence of Tobacco Use among the Adult Lebanese Population. Eastern Mediterranean Health Journal, 7, 819-828.

[19] Ghannem, H. and Fredh, A.H. (1999) Habitudes alimentaires et facteurs de risque cardiovasculaire. La Presse Medicale, 28, 1005-1008.

[20] Centers for Disease Control and Prevention (2005) Global Youth Tobacco Survey: Egypt Fact Sheet.

[21] Centers for Disease Control and Prevention (2003) Global Youth Tobacco Survey: Libya Fact Sheet.

[22] Centers for Disease Control and Prevention (2005) Global Youth Tobacco Survey: Lebanon Fact Sheet.

[23] Bilano, V., Gilmour, S., Moffiet, T., d’Espaignet, E.T., Stevens, G.A., et al. (2015) Global Trends and Projections for Tobacco Use, 1990-2025: An Analysis of Smoking Indicators from the WHO Comprehensive Information Systems for Tobacco Control. The Lancet, 385, 966-976. http://dx.doi.org/10.1016/S0140-6736(15)60264-1 